\title{
Die schwierige Suche nach Ergebnissen der Wahlen zum Europäischen Parlament: Ein neuer Datensatz für die Wahlen 1979 bis 2004*
}

\author{
Markus Tausendpfund und Daniela Braun
}

Seit 1979 werden die Abgeordneten des Europäischen Parlaments (EP) direkt gewählt. Rund 184 Millionen Bürger in neun Mitgliedstaaten waren bei der ersten Direktwahl 1979 wahlberechtigt, bei der sechsten Direktwahl 2004 waren es in 25 Mitgliedstaaten rund 351 Millionen Bürger. Zählte das EP 1979 noch 410 Mitglieder, sind es - nach der Erweiterung der Europäischen Union um Rumänien und Bulgarien im Januar 2007 - mittlerweile 785 Abgeordnete. ${ }^{1}$

Die Einführung direkter Wahlen zum EP - die einzige durch den Bürger direkt legitimierte politische Institution in der EU - sollte das politische Gemeinschaftsgefühl stärken und die politische Unterstützung des europäischen Integrationsprozesses sichern. ${ }^{2}$ Durch die Direktwahl und im Zuge von Vertragsänderungen erhielt das EP einen immer höheren Stellenwert in der europäischen Politik. Damit korrespondiert das Interesse von Wissenschaft und Politik an verlässlichen Ergebnissen der Wahlen zum Europäischen Parlament. ${ }^{3}$ Trotz aller technischen Möglichkeiten gibt es aber Schwierigkeiten bei der Recherche nach amtlichen Europawahlergebnissen. Deshalb soll hier Sekundärliteratur vorgestellt werden, die die Europawahlergebnisse aufführt, wobei Probleme dieser Veröffentlichungen erörtert werden. Vor allem kann dann ein Datensatz vorgestellt werden, der die (amtlichen) Europawahlergebnisse von 1979 bis 2004 enthält.

\section{Entwicklung der Wahlen zum Europäischen Parlament}

Die historischen Wurzeln des EP liegen in der „Gemeinsamen Versammlung“, dem Abgeordnetengremium der im April 1951 gegründeten Europäischen Gemeinschaft für Kohle und Stahl (EGKS). Diese „Gemeinsame Versammlung“ konstituierte sich im September 1952 in Straßburg. Ihre 78 Mitglieder galten als „Vertreter der Völker der in der Gemeinschaft zusammengeschlossenen Staaten “ und wurden von den Parlamenten der sechs Gründungsmitglieder der EGKS entsandt. ${ }^{4}$ Nach der Gründung der Europäischen Wirtschaftsgemeinschaft (EWG) und der Europäischen Atomgemeinschaft (EURATOM) im Jahr 1957 wurde die „Gemeinsame Versammlung“ der EGKS auf alle drei Gemeinschaften ausgedehnt.

* Wir danken Jan van Deth, Hermann Schmitt und Andreas Wüst für wertvolle Kommentare zu früheren Fassungen dieses Artikels.

1 Der Vertrag von Lissabon sieht eine Mitgliederzahl des EP von 750, zuzüglich des Präsidenten, vor (Artikel 9a (2)).

2 Bernhard Weßels / Hermann Schmitt, Europawahlen, Europäisches Parlament und nationalstaatliche Demokratie, in: Hans-Dieter Klingemann / Friedhelm Neidhardt (Hrsg.), Zur Zukunft der Demokratie. Herausforderungen im Zeitalter der Globalisierung, Berlin 2000, S. 295 - 319.

3 Mit verlässlichen Ergebnissen sind in diesem Zusammenhang die amtlichen Ergebnisse der Wahlen zum Europäischen Parlament gemeint.

4 Artikel 21 EGKS. 
Bereits die Gründungsverträge der Europäischen Gemeinschaften sahen die allgemeine und unmittelbare Wahl der Mitglieder des EP nach einem einheitlichen Verfahren in allen Mitgliedstaaten vor. ${ }^{5}$ Allerdings erließ der Ministerrat erst 1976 den Rechtsakt über die Einführung allgemeiner und direkter Wahlen, so dass erstmals 1979 die Abgeordneten des EP in allgemeinen und direkten Wahlen von den Bürgern gewählt werden konnten. ${ }^{6}$

Ein einheitliches Verfahren für die Wahl zum Europäischen Parlament existiert bis heute jedoch nicht. Seit 1979 ist das Wahlrecht im Wesentlichen national geregelt, nur einige Eckpunkte wurden in bisherigen Gemeinschaftsverträgen festgeschrieben. Zu den wenigen Gemeinsamkeiten gehören beispielsweise das aktive Wahlrecht ab 18 Jahren und die Wahl der Abgeordneten auf fünf Jahre. Erst seit 1999 finden in allen Mitgliedsländern die Europawahlen nach Verhältniswahlsystemen statt, zu denen man auch das Verfahren der übertragbaren Einzelstimme (Single Transferable Vote) zählen kann. Der Rat der Europäischen Union hat in seinen Beschlüssen vom 25. Juni und 23. September 2002 das Verhältniswahlsystem auf der Grundlage von Listen oder von übertragbaren Einzelstimmen für die Wahlen zum EP verbindlich festgelegt. Zu den von den Mitgliedsländern frei wählbaren Elementen gehören beispielsweise die Wahlkreiseinteilung, Sperrklauseln (landesweit maximal fünf Prozent) oder verschiedene Methoden der Stimmenverrechnung. ${ }^{7}$ Trotz der ersten Ansätze eines einheitlichen Verfahrens finden die Wahlen zum EP grundsätzlich nach so vielen Wahlsystemen statt wie EU-Mitgliedstaaten ihre Abgeordneten direkt wählen, weshalb Dieter Nohlen von einem "polymorphen Wahlsystem “ 8 spricht. ${ }^{9}$

Der Vertrag von Lissabon schreibt die Wahl der Abgeordneten in allgemeiner, unmittelbarer, freier und geheimer Wahl sowie eine Wahlperiode von fünf Jahren vor. ${ }^{10}$ Danach wie auch bisher - entsprechen die Europawahlen nicht dem Grundsatz einer gleichen Wahl, wonach jede Stimme das gleiche Gewicht haben muss. Durch die in Verträgen festgelegte Mandatskontingentierung wird dieser Grundsatz verletzt. ${ }^{11}$ Das politisch gewollte Missverhältnis der Zahl der Wahlberechtigten und der Anzahl der (nationalen) Abgeordneten führt zu einer Unterrepräsentation der bevölkerungsreicheren und einer Überrepräsentation der bevölkerungsärmeren Staaten im EP. ${ }^{12}$ Dies wird im Vertrag von Lissabon mit dem

5 Artikel 21 (3) EKGS; Artikel 108 (3) EURATOM; Artikel 138 (3) EWG.

6 Amtsblatt der Europäischen Gemeinschaften, 1976, Nr. L 278.

7 Amtsblatt der Europäischen Gemeinschaften, 2002, Nr. L 283/1.

8 Dieter Nohlen, Wie wählt Europa? Das polymorphe Wahlsystem zum Europäischen Parlament, in: APuZ, B 17 (2004), S. 29 - 37.

9 Für weiterführende Informationen zu den nationalen Wahlregeln bei den Europawahlen siehe David M. Farrell / Roger Scully, Electing the European Parliament: How Uniform are ,Uniform' Electoral Systems?, in: Journal of Common Market Studies, 43. Jg. (2005), H. 5, S. 969 - 984; Andreas Haratsch / Christian Koenig / Matthias Pechstein, Europarecht, Tübingen 2006; Andreas M. Wüst / Philip Stöver, Die Wahlsysteme einzelner Länder zur Wahl des Europäischen Parlaments, in: Oskar Niedermayer / Hermann Schmitt (Hrsg.), Europawahl 2004, Wiesbaden 2005, S. $167 \mathrm{ff}$.

10 Vertrag von Lissabon, Artikel 9a (3).

11 Vgl. zu dieser Problematik auch Julia Gieseler, Gewichtetes Wahlrecht: Hilfsmittel für Neugliederungen und angemessene Repräsentation im Bundesstaat?, in: ZParl, 38. Jg. (2007), H. 3, S. 617 -629 , S. $624 \mathrm{f}$.

12 Michael Gallagher / Michael Laver / Peter Mair, Representative government in modern Europe, Boston 2006. 
Begriffspaar „degressiv proportional“ beschrieben, wonach jeder Mitgliedstaat mit mindestens sechs Abgeordneten, aber höchstens 96 Sitzen im EP vertreten ist. ${ }^{13}$

\section{Amtliche Ergebnisse der Wablen zum Europäischen Parlament}

Öffentlichkeit und Wissenschaft haben ein Interesse an verlässlichen Europawahlergebnissen. Letztlich können dies nur die amtlichen Wahlergebnisse sein. Bei den Wahlen zum Europäischen Parlament handelt es sich um nationale Wahlen, und damit sind nationale Institutionen für die Wahldurchführung, die Feststellung des amtlichen Endergebnisses sowie die Veröffentlichung der Wahlergebnisse verantwortlich - in Deutschland zum Beispiel der Bundeswahlleiter, der gleichzeitig auch Präsident des Statistischen Bundesamtes ist. ${ }^{14}$

Diese dezentrale Wahldurchführung hat zur Folge, dass die amtlichen Wahlergebnisse nur über die nationalen Wahlinstitutionen verfügbar sind. Während das Statistische Amt der Europäischen Gemeinschaften (Eurostat) Datenmaterial zu Strukturindikatoren, wirtschaftlicher Entwicklung und öffentlicher Gesundheit in allen EU-Mitgliedstaaten bereithält, gibt es keine europäische Institution, die die amtlichen Wahlergebnisse der einzelnen Länder sammelt und der Öffentlichkeit zur Verfügung stellt. Um die amtlichen Europawahlergebnisse zu erhalten, müssen folglich die einzelnen nationalen Institutionen kontaktiert werden. Als Alternative kommen Sekundärquellen in Frage, die die Wahlergebnisse ausweisen.

\section{Ausgewählte Sekundärquellen zu den Europawahlergebnissen}

Der Großteil der Veröffentlichungen zu den EP-Wahlen konzentriert sich auf jeweils einzelne Länder. Eine Ausnahme stellt die siebte Auflage des Buchs „The European Parliament“ von Richard Corbett, Francis Jacobs und Michael Shackleton dar, welches im Anhang die Ergebnisse der Europawahlen von 1979 bis 2004 nach Ländern ausweist. ${ }^{15}$ Dabei werden die Prozentanteile der nationalen Parteien und die Anzahl der gewählten Abgeordneten pro Partei aufgeführt, es fehlen jedoch die Wahlergebnisse der kleineren Parteien und Angaben zur Wahlbeteiligung. Auch der Sammelband „Dictionnaire des élections européennes“ von Yves Déloye informiert in einzelnen Länderberichten über die nationalen Wahlergebnisse von 1979 bis 2004. ${ }^{16}$ Eine umfangreiche und äußerst gelungene Übersicht, die auch die Resultate der kleineren Parteien berücksichtigt, bietet für 1979 bis 1999 der Sammelband von Pascal Perrineau, Gérard Grunberg und Colette Ysmal. Im Anhang werden die nationalen Wahlergebnisse (absolute Stimmen und Prozentangaben), die Anzahl der gewählten Abgeordneten pro Partei sowie die Wahlbeteiligung aufgeführt. ${ }^{17}$ In einem weiteren Sam-

13 Vertrag von Lissabon, Artikel 9a (2).

14 In einigen europäischen Staaten sind für die Wahldurchführung, die Feststellung des amtlichen Wahlergebnisses sowie die Veröffentlichung der Wahlergebnisse verschiedene Institutionen zuständig. Zum Beispiel stellt in den Niederlanden der Kiesraad das amtliche Wahlergebnis fest, während das Centraal Bureau voor de Statistiek die Ergebnisse veröffentlicht.

15 Richard Corbett / Francis Jacobs / Michael Shackleton, The European Parliament, 7. Auflage, London 2007.

16 Yves Déloye, Dictionnaire des élections européennes, Paris 2005.

17 Laurent de Boissieu / Françoise Chauve / Jean Chiche / Pascal Perrineau / Christian Vandermotten, Appendices: Electoral results by country, groups within the European Parliament, maps, in: Pascal 
melband von Perrineau haben Laurent de Boissieu und Jean Chiche die Ergebnisse der Europawahlen 2004 detailliert zusammengetragen. ${ }^{18}$ Julie Smith listet in einem Anhang die Wahlbeteiligungen und Resultate der Europawahlen bis 1994 auf, ergänzt um die Wahlergebnisse der jüngeren nationalen Hauptwahlen. Allerdings beschränkt sich Smith bei der Darstellung auf die größeren Parteien. ${ }^{19}$

Das Bulletin der Europäischen Union ${ }^{20}$ informiert in knapper Form über die nationalen EP-Wahlergebnisse. Allerdings unterscheiden sich die veröffentlichten Angaben je nach Europawahl. Für die erste finden sich im Bulletin eine kurze Erläuterung der unterschiedlichen nationalen Wahlverfahren, die Wahlbeteiligung der einzelnen Länder und die Sitzverteilung der Fraktionen im EP; allerdings fehlen Informationen zu den Wahlergebnissen der nationalen Parteien. ${ }^{21}$ Die Darstellungen für 1984 und 1989 umfassen die Wahlbeteiligung und die Wahlergebnisse nach Mitgliedstaaten, wobei auch die nationalen Ergebnisse der zurückliegenden Wahlen veröffentlicht wurden. ${ }^{22}$ Zur EP-Wahl 1994 enthält das Bulletin der Europäischen Union die Beteiligungsraten und die nationalen Ergebnisse 1994 und $1989^{23}$, während im Bulletin 1999 lediglich die Wahlbeteiligung in den Mitgliedsländern der Europäischen Union und die Anzahl der gewählten Europaabgeordneten nach nationalen Listen genannt werden. ${ }^{24}$ Für 2004 finden sich weder Angaben zur nationalen Wahlbeteiligung noch Informationen zu den nationalen Wahlergebnissen der Parteien. ${ }^{25}$

Die Forschungsgruppe Wahlen hat zu allen Europawahlen Berichte vorgelegt, die neben den Angaben zur nationalen Wahlbeteiligung auch die Stimmenanteile der nationalen Parteien, die Anzahl der gewählten Europaabgeordneten nach Parteien sowie die Fraktionszuordnung enthalten. Zudem werden die Ergebnisse der jeweils vorangegangenen EP-Wahl und der nationalen Parlamentswahl ausgewiesen. ${ }^{26}$

Perrineau / Gérard Grunberg / Colette Ysmal (Hrsg.), Europe at the polls. The European elections of 1999, New York 2002, S. 239 - 316.

18 Laurent de Boissieu / Jean Chiche, Annexes: Les résultats des élections européennes dans les vingtcinq pays de l'Union européenne, in: Pascal Perrineau (Hrsg.), Le vote européen 2004 - 2005. De l'élargissement au référendum français, Paris 2005, S. 246 - 279.

19 Julie Smith, Europe's Elected Parliament, Sheffield 1999.

20 Das Bulletin der Europäischen Union beschreibt in knapper Form die Maßnahmen der Europäischen Kommission und der übrigen Gemeinschaftsinstitutionen; Herausgeber ist das Generalsekretariat der Europäischen Kommission. Das Bulletin veröffentlicht jedoch nicht die amtlichen Wahlergebnisse.

21 Europäische Kommission, Europawahl: Ergebnisse, in: Bulletin der Europäischen Union (1979), H. 6, S. $20-23$.

22 Europäische Kommission, Europawahlen 1984: Ergebnisse, in: Bulletin der Europäischen Union (1984), H. 6, S. 14 - 18; Europäische Kommission, Europawahlen 1989: Ergebnisse, in: Bulletin der Europäischen Union (1989), H. 6, S. 18 - 22.

23 Europäische Kommission, Wahlen des Europäischen Parlaments 1994: Ergebnisse, in: Bulletin der Europäischen Union (1994), H. 6, S. 132 - 136.

24 Europäische Kommission, Wahlen des Europäischen Parlaments 1999: Ergebnisse, in: Bulletin der Europäischen Union (1999), H. 6, S. 148 ff.

25 Europäische Kommission, Konstituierende Plenarsitzung in Straßburg vom 20. bis 23. Juli 2004, in: Bulletin der Europäischen Union (2004), H. 7/8, S. 127 f.

26 Forschungsgruppe Wahlen, Europawahl in Deutschland. Eine Analyse der 1. Direktwahl zum Europaparlament am 10. Juni 1979, Mannheim 1979; Forschungsgruppe Wahlen, Europawahl. Eine Analyse der 2. Direktwahl zum Europaparlament. 14. bis 17. Juni 1984, Mannheim 1984; Forschungsgruppe Wahlen, Europawahl. Eine Analyse der 3. Direktwahl zum Europaparlament. 15. bis 18. Juni 1989, Mannheim 1989; Forschungsgruppe Wahlen, Europawahl. Eine Analyse 
Ergebnisse der Europawahlen wurden auch in die sechs Sammelbände von Juliet Lodge aufgenommen. Allerdings findet sich ausschließlich im ersten ein ausführlicher Tabellenanhang, während in den späteren Bänden die nationalen Wahlergebnisse in einzelnen Länderberichten dargestellt werden. ${ }^{27}$

Für die ersten drei EP-Wahlen (1979, 1984, 1989) stellen die Veröffentlichungen von Frederick W. S. Craig und Tom T. Mackie aus der Reihe „Europe Votes“ sicherlich die Referenzwerke dar. ${ }^{28}$ Die Bücher informieren nicht nur über die nationalen Wahlergebnisse und Wahlbeteiligungen, sondern schlüsseln diese Daten auch für die regionale Ebene auf. In einem Sammelband hat Mackie die Wahlergebnisse von 1989 und 1994 dokumentiert, allerdings nur auf nationaler Ebene. ${ }^{29}$

Für die Resultate der Wahljahre 1994 und 1999 können die französischsprachigen Veröffentlichungen von Perrineau und Ysmal sowie Grunberg, Perrineau und Ysmal herangezogen werden. ${ }^{30}$ Diese Publikationen listen die nationalen Wahlergebnisse aller EU-Mitgliedsländer auf, für Frankreich zudem die Ergebnisse in den einzelnen départements (für eine Übersicht über alle Sekundärquellen vgl. Tabelle 1).

Die ausgewählten Sekundärveröffentlichungen ${ }^{31}$ weisen zwei Defizite auf. Erstens lassen

der 4. Direktwahl zum Europaparlament. 9. bis 12. Juni 1994, Mannheim 1994; Forschungsgruppe Wahlen, Europawahl 1999. Eine Analyse der Wahl vom 13. Juni 1999, Mannheim 1999; Forschungsgruppe Wahlen, Europawahl 2004. Eine Analyse der Wahl vom 13. Juni 2004, Mannheim 2004.

27 Juliet Lodge / Valentine Herman, Direct elections to the European Parliament: a community perspective, London 1982; Juliet Lodge, Direct elections to the European Parliament 1984, New York 1986; dies., The 1989 election of the European Parliament, Basingstoke 1990; dies., The 1994 elections to the European Parliament, London 1996; dies., The 1999 elections to the European Parliament, Basingstoke 2001; dies., The 2004 Elections to the European Parliament, Basingstoke 2005.

28 Frederick W. S. Craig / Tom T. Mackie, Europe Votes: European parliamentary election results 1979, Chichester 1980; Tom T. Mackie / Frederick W. S. Craig, Europe Votes 2: European parliamentary election results 1979 - 1984, Chichester 1985; Tom T. Mackie, Europe Votes 3: European parliamentary election results 1989, Dartmouth 1990.

29 Tom T. Mackie, Appendix D: The Results of the 1989 and 1994 European Parliament Election, in: Cees van der Eijk / Mark N. Franklin (Hrsg.), Choosing Europe? The European Electorate and National Politics in the Face of Union, Ann Arbor 1996, S. 452 - 471.

30 Pascal Perrineau / Colette Ysmal, Le vote des Douze. Les élections européennes de juin 1994, Paris 1995; Gérard Grunberg / Pascal Perrineau / Colette Ysmal, Le vote des Quinze. Les élections européennes du 13 juin 1999, Paris 2000.

31 Neben den gedruckten Veröffentlichungen informieren auch zahlreiche Internetseiten über die Ergebnisse der Europawahl. Zu den sicherlich am häufigsten genutzten Internetquellen gehören die Seiten des Europäischen Parlaments zur Europawahl 1999 (www.europarl.europa.eu/election/ default.htm) und 2004 (www.europarl.europa.eu/elections2004/ep-election/sites/de/index.html), wobei auf der Internetseite für die Europawahl 2004 darauf hingewiesen wird, dass es sich um provisorische Angaben handelt. Die Norwegian Social Science Data Services bieten eine umfangreiche Datensammlung zu den EP-Wahlen ab 1994 an (http://extweb3.nsd.uib.no/civicactivecms/ opencms/civicactive/en/Data/election/ep/). Die Daten der Internetseite electionworld (www.electionworld.org) sind mittlerweile zur freien Enzyklopädie Wikipedia (www.wikipedia.org) umgezogen, Wahlergebnisse zu den Europawahlen finden sich auch auf der Internetseite Parties and Elections (www.parties-and-elections.de). Grundsätzlich sind die gedruckten Informationen den Internetseiten vorzuziehen, da Informationen auf Internetseiten jederzeit verändert, verlagert oder gelöscht werden können. Zudem besteht insbesondere bei der freien Enzyklopädie Wikipedia die Gefahr, dass jeder, der es möchte, die Informationen verändern kann. 


\begin{tabular}{|c|c|c|c|c|c|c|}
\hline \multicolumn{7}{|c|}{$\begin{array}{l}\text { Tabelle 1: Sekundärquellen zu den Ergebnissen der Wablen zum Europäischen Parlament, } \\
1979 \text { bis } 2004\end{array}$} \\
\hline Veröffentlichung & 1979 & 1984 & 1989 & 1994 & 1999 & 2004 \\
\hline Boissieu / Chauve / Chiche / Perrineau / Vandermotten & $\checkmark$ & $\checkmark$ & $\checkmark$ & $\checkmark$ & $\checkmark$ & \\
\hline Boissieu / Chiche & & & & & & $\checkmark$ \\
\hline Bulletin der Europäischen Kommission & $\checkmark$ & $\checkmark$ & $\checkmark$ & $\checkmark$ & & \\
\hline Corbett / Jacobs / Shackleton & $\checkmark$ & $\checkmark$ & $\checkmark$ & $\checkmark$ & $\checkmark$ & $\checkmark$ \\
\hline Craig / Mackie & $\checkmark$ & & & & & \\
\hline Déloye & $\checkmark$ & $\checkmark$ & $\checkmark$ & $\checkmark$ & $\checkmark$ & $\checkmark$ \\
\hline Forschungsgruppe Wahlen & $\checkmark$ & $\checkmark$ & $\checkmark$ & $\checkmark$ & $\checkmark$ & $\checkmark$ \\
\hline Grundberg / Perrineau / Ysmal & & & & & $\checkmark$ & \\
\hline Lodge & $\checkmark$ & $\checkmark$ & $\checkmark$ & $\checkmark$ & $\checkmark$ & $\checkmark$ \\
\hline Mackie & & & $\checkmark$ & $\checkmark$ & & \\
\hline Mackie / Craig & & $\checkmark$ & & & & \\
\hline Perrineau / Ysmal & & & & $\checkmark$ & & \\
\hline Smith & $\checkmark$ & $\checkmark$ & $\checkmark$ & $\checkmark$ & & \\
\hline \multicolumn{7}{|c|}{$\begin{array}{l}\text { Anmerkung: Die Häkchen zeigen an, zu welchen Europawahlen die Ergebnisse der nationalen Parteien } \\
\text { in den jeweiligen Publikationen zusammengestellt wurden. Die Literaturangaben zu den aufgeführten } \\
\text { Sekundärquellen sind in den Fußnoten } 15 \text { bis } 19 \text { sowie } 21 \text { bis } 30 \text { vermerkt. } \\
\text { Quelle: Eigene Zusammenstellung. }\end{array}$} \\
\hline
\end{tabular}

sich immer wieder Unterschiede zwischen den dort veröffentlichten Wahlergebnissen feststellen. So variiert beispielsweise der Prozentanteil der Konservativen in Großbritannien bei der Europawahl 1994 zwischen 26,8 Prozent und 27,9 Prozent - je nach Veröffentlichung. Weist die Forschungsgruppe Wahlen für die Liberalen in Luxemburg für 1999 einen Stimmenanteil von 20,8 Prozent aus ${ }^{32}$, erhält dieselbe Partei bei Corbett, Jacobs und Shackleton 20 Prozent $^{33}$ und bei Boissieu, Françoise Chauve, Chiche, Perrineau und Christian Vandermotten 20,5 Prozent. ${ }^{34}$ Und als drittes Beispiel für kleine, aber feine Unterschiede sei auf das Wahlergebnis der Sozialisten in Portugal bei der EP-Wahl 1994 verwiesen: Bei Mackie werden 36 Prozent angegeben ${ }^{35}$, das Bulletin der Europäischen Union vermeldet 34,8 Prozent. ${ }^{36}$ Neben solchen Unterschieden in den veröffentlichten Wahlergebnissen wird zweitens in den seltensten Fällen eine konkrete Quelle für die aufgeführten Daten genannt.

Diese beiden Mängel waren Anlass, einen Datensatz zu den Ergebnissen der Europawahlen von 1979 bis 2004 zu erstellen, der nahezu ausschließlich auf den amtlichen Wahlergebnissen der jeweils national zuständigen Institution beruht.

32 Forschungsgruppe Wahlen, Europawahl 1999. Eine Analyse der Wahl vom 13. Juni 1999, Mannheim 1999, S. C17.

33 Richard Corbett / Francis Jacobs / Michael Shackleton, a.a.O., S. 361.

34 Laurent de Boissieu / Françoise Chauve / Jean Chiche / Pascal Perrineau / Christian Vandermotten, a.a.O., S. $239-316$.

35 Tom T. Mackie, Appendix D, a.a.O., S. 452 - 471.

36 Europäische Kommission, Wahlen des Europäischen Parlaments 1994, a.a.O. 


\section{Auf der Suche nach amtlichen Ergebnissen der Wablen zum EP}

Die amtlichen Europawahlergebnisse werden nicht, wie bereits geschildert, von einer europäischen Institution gesammelt und publiziert. Deshalb wurden in einem ersten Schritt die nationalen Institutionen, die die amtlichen Wahlergebnisse feststellen beziehungsweise publizieren, ermittelt. Dabei zeigte sich, dass in den 25 untersuchten Mitgliedstaaten der Europäischen Union drei unterschiedliche Institutionen für die Feststellung und Veröffentlichung der amtlichen Europawahlergebnisse zuständig sind: erstens die Regierung des jeweiligen Mitgliedstaats, wobei zumeist ein bestimmtes Ministerium verantwortlich ist; zweitens ein nationales Wahlkomitee oder drittens das nationale Statistikbüro. Um die Stimmenanteile der nationalen Parteien aller Mitgliedsländer und Wahljahre zu ermitteln, wurde mit den jeweiligen Wahlbehörden Kontakt aufgenommen. Auf diese Weise konnten in den überwiegenden Fällen die amtlichen Ergebnisse für die einzelnen Mitgliedsländer der bisherigen sechs EP-Wahlen ermittelt werden. Eine Übersicht bietet Tabelle 2, in der auch vermerkt ist, ob die amtlichen Wahlergebnisse in den Datensatz aufgenommen wurden.

\begin{tabular}{|c|c|c|c|}
\hline Land & Nationale Wahlbehörde & Name der Institution & $\begin{array}{l}\text { Amtliches } \\
\text { Ergebnis in } \\
\text { Datensatz } \\
\text { aufgenom- } \\
\text { men? }\end{array}$ \\
\hline Belgien & Regierung & $\begin{array}{l}\text { Directie van de Verkiezingen; } \\
\text { Direction des Elections }\end{array}$ & ja \\
\hline Dänemark & Regierung & Indenrigs- og Sundhedsministeriet & ja \\
\hline Deutschland & Nationales Amt für Statistik & Bundeswahlleiter & ja \\
\hline Estland & Nationales Wahlkomitee & Vabariigi Valimiskomisjon & ja \\
\hline Finnland & $\begin{array}{l}\text { Nationales Amt für Statistik } \\
(1979-1994) \\
\text { Regierung }(1999-2004) \\
\end{array}$ & $\begin{array}{l}\text { Tilastokeskus } \\
\text { Oikeusministeriö } \\
\end{array}$ & ja \\
\hline Frankreich & Regierung & Ministère de l'Intérieur & ja \\
\hline Griechenland & Regierung & Ministry of Interior Affairs & teilweise $^{a}$ \\
\hline Großbritannien & $\begin{array}{l}\text { Nationales Wahlkomitee } \\
\text { (seit 2004) }\end{array}$ & The Electoral Commission & teilweise $^{\mathrm{b}}$ \\
\hline Irland & Regierung & $\begin{array}{l}\text { Department of the Environment, } \\
\text { Heritage and Local Government }\end{array}$ & ja \\
\hline Italien & Regierung & Ministero dell'Interno & teilweise $^{\mathrm{c}}$ \\
\hline Lettland & Nationales Wahlkomitee & Centrālā vēlēšanu komisija & ja \\
\hline Litauen & Nationales Wahlkomitee & Vyriausioji rinkimų komisija & ja \\
\hline Luxemburg & Nationales Amt für Statistik & $\begin{array}{l}\text { Service central de la statistique } \\
\text { et des études économiques }\end{array}$ & ja \\
\hline Malta & Regierung & Department of Information & ja \\
\hline Niederlande & Nationales Amt für Statistik & Centraal Bureau voor Statistiek & ja \\
\hline Österreich & Regierung & Bundesministerium für Inneres & ja \\
\hline Polen & Nationales Wahlkomitee & Państwowa Komisja Wyborcza & ja \\
\hline Portugal & Nationales Wahlkomitee & Comissão Nacional de Eleiçóes & ja \\
\hline Schweden & Nationales Amt für Statistik & Valmyndigheten & ja \\
\hline
\end{tabular}




\begin{tabular}{|c|c|c|c|}
\hline \multicolumn{4}{|c|}{ FortsetzungTabelle 2} \\
\hline Slowakei & Nationales Amt für Statistik & Štatistický úrad slovenskej republiky & ja \\
\hline Slowenien & Regierung & Vlada Republike Slovenije & ja \\
\hline Spanien & Regierung & Ministerio del Interior & ja \\
\hline Tschechien & Nationales Wahlkomitee & Český statistický úrad & ja \\
\hline Ungarn & Nationales Wahlkomitee & Országos Választási Iroda & ja \\
\hline Zypern & Regierung & Ministry of the Interior & nein $^{\mathrm{d}}$ \\
\hline \multicolumn{4}{|c|}{$\begin{array}{l}\text { Anmerkungen: } \\
\text { a Für Griechenland werden für die Jahre } 1979 \text { bis } 1999 \text { Ergebnisse ausgewiesen, die die Leiterin der Eu- } \\
\text { ropawahlstudie } 2004 \text { für Griechenland, Eftichia Teperoglou, zur Verfügung stellte. } \\
\text { b In Großbritannien war zwischen } 1979 \text { und } 1999 \text { keine offizielle Institution für die Veröffentlichung der } \\
\text { amtlichen Europawahlergebnisse zuständig. Für diesen Zeitraum wurden die Ergebnisse der House of } \\
\text { Commons Library verwendet. } \\
\text { c Für Italien wurde für die Wahlergebnisse von } 1979 \text { bis } 1989 \text { auf eine Veröffentlichung des nationalen } \\
\text { Amts für Statistik (Osservatorio elettorale) zurückgegriffen. } \\
\text { d Die zyprischen Ergebnisse sind der Tageszeitung Ta Nea vom 14. Juli } 2004 \text { entnommen, da das Minis- } \\
\text { terium nicht auf Anfragen reagiert hat. } \\
\text { Quelle: Eigene Darstellung. }\end{array}$} \\
\hline
\end{tabular}

\section{Mannheimer Dokumentation der amtlichen Europawablergebnisse 1979 bis 2004}

Auf der Basis dieser Recherchen konnte die Mannheimer Dokumentation der amtlichen Europawahlergebnisse erstellt werden. Sie enthält nunmehr die amtlichen Ergebnisse der Wahlen zum Europäischen Parlament, die von den nationalen Wahlbehörden übermittelt wurden. Der Datensatz wurde noch um einige Merkmale erweitert und steht zusammen mit einer Datensatzbeschreibung auf der Homepage des Mannheimer Zentrums für Europäische Sozialforschung (MZES) zur Verfügung. ${ }^{37}$

Im Datensatz sind grundlegende Informationen enthalten: Das Jahr, in dem die bisherigen Wahlen stattfanden, die Mitgliedsländer der EU, in denen die Parteien gewählt wurden, sowie die Angabe, zu welchem Zeitpunkt in den jeweiligen Mitgliedsländern erstmals eine Wahl zum Europäischen Parlament durchgeführt wurde. Insgesamt werden 25 Mitgliedsländer dokumentiert (Rumänien und Bulgarien sind erst seit 1. Januar 2007 Mitglied in der EU und sind daher noch nicht berücksichtigt). Der Datensatz enthält die Ergebnisse für Großbritannien und Nordirland, so dass Stimmenanteile und Anzahl der Sitze jeweils

37 Dieser Datensatz ist im Rahmen des von der Deutschen Forschungsgemeinschaft geförderten Euromanifesto-Projekts am Mannheimer Zentrum für Europäische Sozialforschung (MZES) entstanden und steht auf der MZES-Homepage (http://www.mzes.uni-mannheim.de/fs_daten_d. html) mit einem ausführlichen Kodebuch zur Verfügung. Das Euromanifesto-Projekt wurde von Hermann Schmitt geleitet und von Andreas Wüst gemanagt. Für eine Beschreibung des Euromanifesto-Projekts Andreas M. Wüst / Hermann Schmitt, Comparing the Views of Parties and Voters in the 1999 Election to the European Parliament, in: Cees van der Eijk / Wouter van der Brug (Hrsg.), European Elections \& Domestic Politics: Lessons from the Past and Scenarios for the Future, Notre Dame 2007, S. 73 - 93. Für eine detaillierte Beschreibung des Kodierschemas siehe Andreas M. Wüst / Andrea Volkens, Euromanifesto Coding Instructions, Arbeitspapier 64, Mannheim 2003. 


\begin{tabular}{|l|l|}
\hline \multicolumn{2}{|l|}{ Tabelle 3: Informationen in der Mannheimer Dokumentation der amtlichen Europawabl- } \\
ergebnisse, 1979 bis 2004
\end{tabular}

gesondert für die beiden Gebiete ausgewiesen werden. Weitere Details sind Tabelle 3 zu entnehmen.

\section{Zur Notwendigkeit einer europäischen Institution für die Dokumentation der Wahlergebnisse}

Zwar handelt es sich auch bei der Mannheimer Dokumentation der Europawahlergebnisse um eine Sekundärquelle. Gegenüber zahlreichen anderen Veröffentlichungen sind dort aber die verwendeten Quellen sorgfältig dokumentiert und im Datensatz enthalten. Die aufgeführten Wahlergebnisse können demnach grundsätzlich auf ihre Korrektheit überprüft werden.

Wissenschaft und Öffentlichkeit haben ein berechtigtes Interesse an einem einfachen und unmittelbaren Zugang zu den amtlichen Europawahlergebnissen, deren Bereitstellung derzeit aber nur in unzureichendem Maße erfolgt. Interessierte Personen müssen in der Regel auf Sekundärquellen ausweichen, um die gewünschten Informationen zu erhalten. $\mathrm{Zu}$ lösen ist dieses Kollektivgutproblem nur durch einen entsprechenden Beschluss des Europäischen Parlaments beziehungsweise der Europäischen Kommission. Diese Institutionen verfügen über die notwendigen Mittel, um dem Bedarf von Öffentlichkeit und Wissenschaft nach verlässlichen Wahlergebnissen Rechnung zu tragen. 
Insbesondere mit Blick auf die Europawahl 2009 scheint es geboten, eine (europäische) Institution zu beauftragen, die die amtlichen Ergebnisse der EP-Wahlen sammelt, auswertet und zur Verfügung stellt. Die nationalen Ergebnisse und Beteiligungen der Wahlen zum Europäischen Parlament sind dabei der Ausgangspunkt. Wünschenswert sind insbesondere auch regionale / lokale Wahlergebnisse, um Abweichungen vom nationalen Trend feststellen zu können. Zudem sind die Resultate der nationalen Hauptwahlen zu berücksichtigen, da die Europawahlergebnisse am besten als Abweichung von diesen interpretiert werden können. Angaben zum Wahlsystem und Wahlverfahren (Wahlpflicht, Wahltermin) sowie die Information, ob die EP-Wahl mit der nationalen Hauptwahl oder mit einer anderen, wichtigen Nebenwahl zusammenfällt, würden zudem eine erste Einordnung der Ergebnisse und Beteiligungsraten im internationalen Vergleich ermöglichen.

\title{
Ökonomisches Wählen: Zum Einfluss von Wahrnehmungen der allgemeinen Wirtschaftslage auf das Abschneiden der Bundesregierungsparteien bei Landtagswahlen*
}

\author{
Ivar Krumpal und Adrian Vatter
}

Politikwissenschaftler waren seit jeher an der engen Verflechtung zwischen Wirtschaft und Politik interessiert. Entsprechend häufig werden diese komplexen Zusammenhänge untersucht. Insbesondere im Bereich der empirischen Wahlforschung haben sich auf dem Gebiet des ökonomischen Wählens („Economic Voting“) recht robuste Trends auf der Aggregatebene abgezeichnet. Dabei verdichten sich die Befunde zu einem relativ stabilen Zusammenhang zwischen der allgemeinen Konjunkturentwicklung eines Landes und der Popularitäts- beziehungsweise Stimmentwicklung der jeweils amtierenden Regierungsparteien. ${ }^{1}$ Demnach ist mit überproportional hohen Stimmenverlusten besonders dann zu rechnen, wenn zum Zeitpunkt einer Wahl die wirtschaftliche Entwicklung negativ ist.

Allerdings zeichnen sich diese auf Aggregatdaten beruhenden Untersuchungen für Deutschland durch theoretische und methodische Schwächen aus. So fehlt ihnen in der Regel eine mikrotheoretische Fundierung ihrer Hypothesen, und die empirischen Ergebnisse unterliegen der bekannten Gefahr eines ökologischen Fehlschlusses. Die bisherigen Aggregatdatenstudien zum ökonomischen Wählen enthalten meist nur implizite Annahmen darüber, wie der einzelne Wähler seine subjektive wirtschaftliche Situation konkret wahrnimmt und welches Entscheidungsverhalten daraus resultiert. Zudem wird vernachläs-

* Für zahlreiche konstruktive Diskussionen und kritische Anmerkungen danken wir Heiko Rauhut, Thomas Voss und Wolfgang Langer. Der Beitrag ist teilweise im Rahmen des von der DFG geförderten Forschungsprojekts „Demokratiemuster in den deutschen Bundesländern“ entstanden, das von Markus Freitag und Adrian Vatter bearbeitet wird.

1 Michael S. Lewis-Beck / Mary Stegmaier, Economic Determinants of Electoral Outcomes, in: Annual Review of Political Science, 3. Jg. (2000), S. 183 - 219; Brian J. Gaines / Christophe Crombez, Another Look at Connections Across German Elections, in: Journal of Theoretical Politics, 16. Jg. (2004), S. $289-319$. 